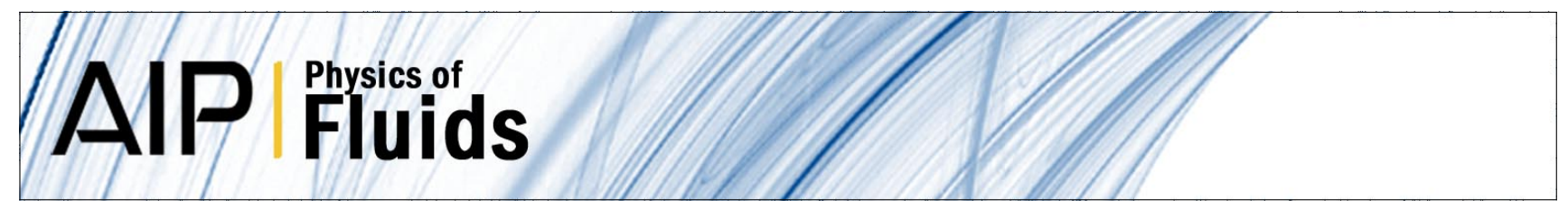

\title{
Transport processes in random arrays of cylinders. I. Thermal conduction
}

\author{
A. S. Sangani and C. Yao
}

Citation: Phys. Fluids 31, 2426 (1988); doi: 10.1063/1.866595

View online: http://dx.doi.org/10.1063/1.866595

View Table of Contents: http://pof.aip.org/resource/1/PFLDAS/v31/i9

Published by the American Institute of Physics.

\section{Related Articles}

Rheological, optical, and thermal characterization of temperature-induced transitions in liquid crystal ferrosuspensions

J. Appl. Phys. 111, 07B308 (2012)

Effects of bending and torsion rigidity on deformation and breakage of flexible fibers: A direct simulation study J. Chem. Phys. 136, 074903 (2012)

Investigations of field instability of ferrofluid in hypergravity and microgravity

AlP Advances 2, 012138 (2012)

Shear-induced particle migration and margination in a cellular suspension

Phys. Fluids 24, 011902 (2012)

Influence of structure in heterodyne electrophoretic light scattering

J. Chem. Phys. 136, 044201 (2012)

\section{Additional information on Phys. Fluids}

Journal Homepage: http://pof.aip.org/

Journal Information: http://pof.aip.org/about/about_the_journal

Top downloads: http://pof.aip.org/features/most_downloaded

Information for Authors: http://pof.aip.org/authors

\section{ADVERTISEMENT}

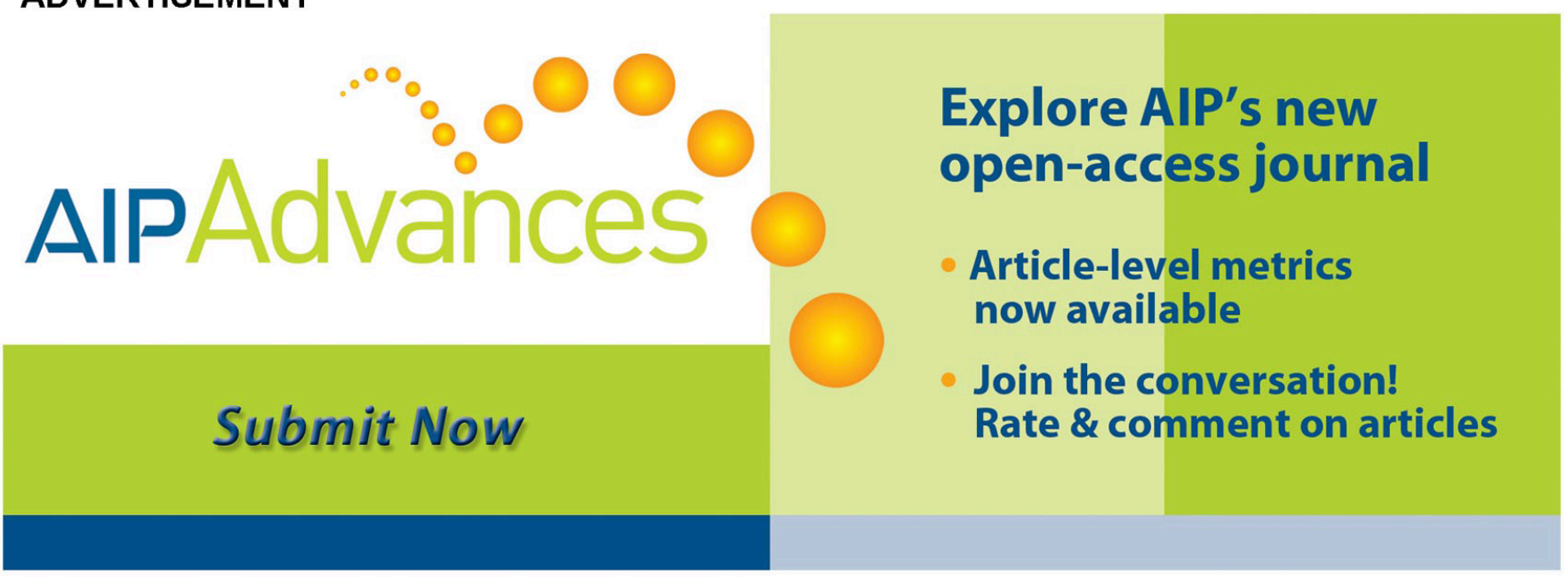




\title{
Transport processes in random arrays of cylinders. I. Thermal conduction
}

\author{
A. S. Sangani and C. Yao \\ Department of Chemical Engineering and Materials Science, Syracuse University, Syracuse, New York \\ 13244-1190
}

(Received 6 May 1987; accepted 31 May 1988)

\begin{abstract}
A numerical method is developed that takes into account the many-particle interactions in a rigorous manner to determine the effective thermal conductivity $K_{m}$ of a composite medium consisting of parallel circular cylinders of thermal conductivity $\alpha k$ suspended in a matrix of conductivity $k$. Numerical results for $K_{m}$ are presented for a wide range of $\alpha$ and $\phi$, the area fraction of the cylinders, after averaging over several computer-generated random arrays of cylinders. The results obtained via this exact method are compared with those of various approximate analytical methods to assess their utility in predicting $K_{m}$.
\end{abstract}

\section{INTRODUCTION}

Transport of mass, momentum, or energy in heterogeneous media consisting of particles suspended in a fluid or another solid medium plays an important role in a wide variety of physical operations. Consequently, there have been numerous studies in the past whose goal is to provide a suitable framework for describing quantitatively the transport processes in such systems. A large number of these studies involve approximations that are often ad hoc in nature. Rigorous treatments are available for relatively simple transport processes in relatively simple systems such as those in which either the volume fraction of the particles is very small compared to unity or in which the transport properties of the particles are not too different from those of the surrounding medium. Two exceptions to this are the expressions for the bounds on various macroscopic transport coefficients and the exact results for a few selected spatially periodic arrangements of the particles. Unfortunately, the former gives bounds that require rather tedious computations of the higher-order moments of the spatial distribution of the particles, whereas the latter is sometimes criticized for its highly idealized spatial distribution.

The main objective of this series of two papers is to illustrate a numerical method for evaluating the macroscopic transport coefficients by computing exactly the many-particle interactions in systems with an arbitrary size and spatial distribution of the cylindrical particles. In this paper, the problem of determining the macroscopic or effective thermal conductivity of the suspensions is considered; that of determining the permeability or, equivalently, the average pressure gradient in a viscous fluid steadily moving through an assemblage of fixed particles is treated in the second paper. In addition to providing the exact results for the above transport coefficients for a few realistic spatial distributions of the particles, the method can also be used to obtain detailed temperature and velocity distributions that may prove valuable in the study of more complex transport processes such as the filtration of aerosols by fibrous materials.

The organization of the paper is as follows. In Sec. II, an exact method is described for determining the temperature field around $N$ interacting parallel cylinders in a periodic domain. This method is applied to several computer-generated random arrays in Sec. III to determine the average mac- roscopic or effective thermal conductivity of such arrays as a function of $\phi$, the area fraction of the cylinders. In Sec. IV, these results are compared with the predictions of various approximate methods, asymptotic expressions, and bounds on the effective conductivity to assess the range of usefulness/validity of these methods, at least as far as their application to the random arrays of cylinders is concerned. In particular a method is described for determining rigorously the higher-order bounds on the thermal conductivity of arrays of cylinders, and using this method a constant appearing in the third-order bounds is evaluated and compared with an approximate method suggested in the literature to evaluate the same bound. The predictions of the approximate methods are also compared with the exact results for the periodic arrays of cylinders.

\section{THE METHOD}

\section{A. An exact solution for the temperature field for $N$ interacting particles in a periodic domain}

Consider a composite medium consisting of infinitely long parallel cylinders of thermal conductivity $\alpha k$ suspended in a substance whose thermal conductivity is $k$. The medium is spatially periodic with its unit cell of dimensions $h \times h$, and the centers of the particles within a representative unit cell are given by

$$
\mathbf{r}=\mathbf{R}^{(n)}, \quad n=1,2, \ldots, N .
$$

The radius of the $n$th particle is $a^{(n)}$.

We wish to determine the temperature field resulting from heat conduction when a temperature gradient is externally imposed on the above composite medium. Since this field satisfies the Laplace equation, a general expression for the temperature at any point in the matrix can be written in terms of $S_{1}$, the periodic singular solution of the Laplace equation, as follows. As shown by Hasimoto,'

$$
S_{1}(\mathbf{r})=\frac{1}{\pi h^{2}} \sum_{\mathbf{k}_{\mathbf{L}} \neq 0} k_{\mathbf{L}}^{-2} \exp 2 \pi i \mathbf{k}_{\mathbf{L}} \cdot \mathbf{r}
$$

satisfies

$$
\nabla^{2} S_{1}=4 \pi\left(\frac{1}{h^{2}}-\sum_{\mathbf{r}_{\mathbf{L}}} \delta\left(\mathbf{r}-\mathbf{r}_{\mathbf{L}}\right)\right),
$$

where 


$$
\begin{aligned}
& \mathbf{r}_{\mathbf{L}}=h\left(l_{1} \mathbf{e}_{1}+l_{2} \mathbf{e}_{2}\right), \\
& \mathbf{k}_{\mathbf{L}}=h^{-1}\left(l_{1} \mathbf{e}_{1}+l_{2} \mathbf{e}_{2}\right), \quad l_{1}, l_{2}=0, \pm 1, \pm 2, \ldots,
\end{aligned}
$$

and $e_{1}$ and $e_{2}$ are unit vectors along the $x_{1}$ and $x_{2}$ axes. Although $S_{1}$ itself does not satisfy the Laplace equation, its derivatives do, and hence the temperature can be expressed in terms of the derivatives of $S_{1}$ as

$$
\begin{aligned}
T(r)= & \mathbf{G} \cdot \mathbf{x}+\sum_{n=1}^{N} \sum_{l=1}^{\infty} \sum_{m=0}^{1} A_{l m}^{(n)} \\
& \times\left(\frac{\partial}{\partial x_{1}}\right)^{l-m}\left(\frac{\partial}{\partial x_{2}}\right)^{m} S_{1}\left(\mathbf{r}-\mathbf{R}^{(n)}\right),
\end{aligned}
$$

where $\mathbf{G}$ is the imposed or the mean temperature gradient in the composite medium. Note that the derivatives of $S_{1}$ with order 2 or greater in $x_{2}$ can be expressed as a linear combination of the terms already included in (5) by making use of (3), and hence (5) represents the most general expression for the temperature at any point in the matrix.

To complete the solution for the temperature field, we must determine the unknown coefficients $\boldsymbol{A}_{l m}$ appearing in (5). This is done by applying the condition of the continuity of temperature and flux at the surface of each of the $N$ particles. We first express the temperature inside the $n$th particle in terms of the polar coordinates $(s, \theta)$ with respect to its center as

$$
\begin{aligned}
T^{(n)}(\mathbf{r})= & \sum_{p=0}^{\infty}\left(E_{p}^{(n)} s^{p} \cos p \theta\right. \\
& \left.+\widetilde{E}_{p}^{(n)} s^{p} \sin p \theta\right), \text { for } s \leqslant Q^{(n)},
\end{aligned}
$$

where $s=\left|\mathbf{r}-\mathbf{R}^{(n)}\right|, \theta$ is the angle between the vector $\mathbf{r}-\mathbf{R}^{(n)}$ and the $x_{1}$ axis, and $E_{p}^{(n)}$ and $\widetilde{E}_{\rho}^{(n)}$ are additional unknown coefficients. Similarly, the temperature field just outside the $n$th particle can be expressed as

$$
\begin{aligned}
T^{(n)}(\mathbf{r})= & C_{0}^{(n)}+\sum_{p=1}^{\infty}\left(C_{p}^{(n)} s^{p}+D_{p}^{(n)} s^{-p}\right) \cos p \theta \\
& +\left(\tilde{C}_{p}^{(n)} s^{p}+\tilde{D}_{p}^{(n)} s^{-p}\right) \sin p \theta, \text { for } s \geqslant a^{(n)},
\end{aligned}
$$

where $C_{p}^{(n)}, D_{p}^{(n)}, \tilde{C}_{p}^{(n)}$, and $\tilde{D}_{p}^{(n)}$ are related to $A_{l m}$. gives

The continuity of the temperature and flux at $s=a^{(n)}$

$$
\beta C_{p}^{(n)}=-D_{p}^{(n)}\left(a^{(n)}\right)^{-2 p}, \quad \beta \equiv(\alpha-1) /(\alpha+1),
$$

plus a similar relationship between $\tilde{C}_{p}$ and $\tilde{D}_{p}$. Now to relate the coefficients $D_{p}$ and $\tilde{D}_{p}$ to $A_{l m}$, we use the following expansion of $S_{1}$ near $s=0$ (see Ref. 2):

$S_{1}(s)=-2 \log \frac{s}{h}-\bar{c}+\frac{\pi s^{2}}{h^{2}}+\sum_{n=1}^{\infty} c_{2 n} s^{4 n} \cos 4 n \theta$,

where $\bar{c}=2.6232$ and $c_{2 n} h^{4 n}$ are related to Rayleigh's ${ }^{3}$ sums $\Sigma_{4 n}$ by

$$
c_{2 n} h^{4 n}=\Sigma_{4 n} / 2 n \text {. }
$$

The numerical values for Rayleigh's sums are reported in Ref. 4. Thus all the constants appearing in (8) are known, and $S_{1}$ can be eyaluated to a sufficiently high accuracy at any point inside the unit cell containing $s=0$.

Since the singular terms $\left(s^{-p}\right)$ in (6b) can result only from the differentiation of the logarithmic term in (8), we find that

$$
\begin{aligned}
& D_{p}^{(n)}=-(n ! / 2) A_{p 0}^{(n)}, \\
& \tilde{D}_{p}^{(n)}=-(n ! / 2) A_{p 1}^{(n)} .
\end{aligned}
$$

On the other hand, the growing harmonics in (6b) arise upon differentiating the nonsingular part of $S_{1}\left(\mathbf{r}-\mathbf{r}^{(m)}\right)$, $m=1,2, \ldots, N$. The contribution due to the $n$th particle can be directly evaluated from (8) whereas that from any other particle can be evaluated by employing the following theorem for the Laplace functions.

Theorem: Let $\psi$ be a solution of the Laplace equation and regular in some domain $D$ containing $\mathbf{R}^{(n)}$. Then for any point in $D, \psi$ can be expressed as

$$
\begin{gathered}
\psi(\mathbf{r})=\sum_{p=0}^{\infty}\left(\psi_{p} s^{p} \cos p \theta+\tilde{\psi}_{p} s^{p} \sin p \theta\right), \\
s=\left|\mathbf{r}-\mathbf{R}^{(n)}\right|,
\end{gathered}
$$

where

$$
\begin{aligned}
& \psi_{P}=\frac{1}{p !}\left(\frac{\partial}{\partial x_{1}}\right)^{p} \psi\left(\mathbf{R}^{(n)}\right), \\
& \tilde{\psi}_{p}=-\frac{1}{p !}\left(\frac{\partial}{\partial x_{1}}\right)^{p-1} \frac{\partial}{\partial x_{2}} \psi\left(\mathbf{R}^{(n)}\right) .
\end{aligned}
$$

The proof of the above theorem is quite straightforward. On making use of the above theorem together with (5), (7), and (10), we finally arrive at the following set of linear equations relating the unknowns $A_{l m}$ :

$$
\begin{aligned}
A_{l 0}^{(n)}= & \frac{-(-1)^{\prime} \beta\left(a^{(n)}\right)^{l}}{2(l-1) ! l !}\left\{\sum_{\substack{m=1 \\
m \neq n}}^{N} \sum_{p=1}^{\infty}\left[A_{p 0}^{(m)}\left(\frac{\partial}{\partial x_{1}}\right)^{l+p}+A_{p 1}^{(m)}\left(\frac{\partial}{\partial x_{1}}\right)^{l+p-1}\left(\frac{\partial}{\partial x_{2}}\right)\right]\right. \\
& \left.\cdot S_{1}\left(\mathbf{R}^{(n)}-\mathbf{R}^{(m)}\right)+A_{p 0}^{(n)} c_{q}(l+p) !+\delta_{l 1}\left(G_{1}+\frac{2 \pi}{h^{2}} A_{10}^{(n)}\right)\right\}, \\
A_{l 1}^{(n)}= & \frac{-(-1)^{l} \beta\left(a^{(n)}\right)^{2 l}}{2(l-1) ! l !}\left\{\sum_{\substack{m=1 \\
m \neq n}}^{N} \sum_{p=1}^{\infty}\left[A_{p 0}^{(m)}\left(\frac{\partial}{\partial x_{1}}\right)^{l+p-1} \frac{\partial}{\partial x_{2}}+A_{p 1}^{(m)}\left(\frac{\partial}{\partial x_{1}}\right)^{l+p-2}\left(\frac{\partial}{\partial x_{2}}\right)^{2}\right]\right. \\
& \left.\cdot S_{1}\left(\mathbf{R}_{!}^{(n)}-\mathbf{R}^{(m)}\right)-A_{p 1}^{(n)} c_{q}(l+p) !+\delta_{l 1}\left(G_{2}+\frac{2 \pi}{h^{2}} A_{11}^{(n)}\right)\right\},
\end{aligned}
$$

where 
$c_{q}=\left\{\begin{array}{l}c_{(l+p) / 2}, \text { if }(l+p) / 4 \text { is an integer, } \\ 0, \text { otherwise, }\end{array}\right.$

and $G_{1}$ and $G_{2}$ are the components of $G$ along the $x_{1}$ and $x_{2}$ axes. We note that the above equations contain various derivatives of $S_{1}$ to be evaluated at the vector difference $\mathbf{R}^{(n)}-\mathbf{R}^{(m)}$. These quantities are computed by differentiating each term in (8) separately and adding the result. The formulas required for this purpose are collected in the Appendix. The series computed from (8) diverges for higherorder derivatives and for $s=\left|\mathbf{R}^{(m)}-\mathbf{R}^{(n)}\right|>h / 2$. An alternate expression is therefore needed to evaluate the higher-order derivatives of $S_{1}$ for $s>h / 2$. This expression is also given in the Appendix.

This completes the description of the formal solution for the temperature field around $N$ interacting cylinders in a periodic domain. The solution given by (5), (13), and (14) can be used either to compute the macroscopic or effective thermal conductivity of the composites which are periodic with each unit cell containing $N$ particles or to study the temperature field around a finite number of particles in an infinite medium provided that there is no net source or sink of heat in the medium. In the latter case, $h=\infty$ and, in lieu of (8), the derivatives of $S_{1}$ may be computed by taking $S_{1}=-2 \log s$. In the present study, we shall be interested in the determination of the effective conductivity of the composites.

\section{B. Effective thermal conductivity}

The mean heat flux in the composite medium described in Sec. II $\mathrm{A}$ is proportional to the mean temperature gradient $G_{1}$, and the proportionality is known as the effective conductivity of the composite. In general, for an arbitrary choice of $\mathbf{R}^{(n)}$, the medium is anisotropic and the conductivity is a second-order tensor $k_{i j}^{*}$ as given by

$$
\left\langle q_{i}\right\rangle=-k_{i j}^{*} G_{j},
$$

with $k_{i j}^{*}=k_{j i}^{*}$. Here, \langle\rangle denotes the average over a unit cell. The average heat flux can be calculated as follows. We decompose $\left\langle q_{i}\right\rangle$ to

$$
\begin{aligned}
h^{2}\left\langle q_{i}\right\rangle & =-k \int_{V_{m}} \frac{\partial T}{\partial x_{i}} d V-\alpha k \sum_{n=1}^{N} \int_{V_{n}} \frac{\partial T}{\partial x_{i}} d V \\
& =-k\left(G_{i} h^{2}+(\alpha-1) \sum_{n=1}^{N} \int_{\partial V_{n}} T n_{i} d A\right),
\end{aligned}
$$

where $V_{m}$ denotes the region occupied by the matrix, $V_{n}$ denotes the region occupied by the $n$th cylinder whose surface is $\partial V_{n}$, and $n_{i}$ is the unit outward normal vector on the surface $\partial V_{n}$.

The integral in (17) is related to the coefficients of $\sin \theta$ and $\cos \theta$ in the expansion of $T$ near the center of $n$th particle, and hence it can be readily related to the constants $A_{10}$ and $A_{11}$ by making use of (6), (7), and (10). For example,

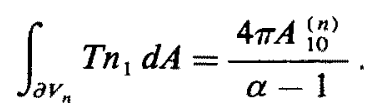

On substituting (18) into (17), we obtain

$$
k_{11} G_{1}+k_{12} G_{2}=G_{1}+\frac{4 \pi}{h^{2}} \sum_{n=1}^{N} A_{10}^{(n)},
$$

$$
k_{21} G_{1}+k_{22} G_{2}=G_{2}+\frac{4 \pi}{h^{2}} \sum_{n=1}^{N} A_{11}^{(n)} \quad\left(k_{i j} \equiv k k_{i j}^{*}\right)
$$

Thus, in order to determine all the components of the effective conductivity tensor, we need to solve only two problems. In the first problem, $G_{1}=1$ and $G_{2}=0$, i.e., the mean temperature gradient is along the $x_{1}$ axis. The solution of this problem yields $k_{11}$ and $k_{21}$. In the second problem, $G_{1}=0$ and $G_{2}=1$, the solution of which determines $k_{12}$ and $k_{22}$. Of course, if the temperature gradient is in the $x_{3}$ direction, i.e., parallel to the axes of the cylinders, then all $A_{l m}$ are identically zero and $k_{i 3}$ is then trivially determined from

$$
k_{i 3}=[1+(\alpha-1) \phi] \delta_{i 3},
$$

where the area fraction $\phi$ is given by

$$
\phi=\frac{\pi}{h^{2}} \sum_{n=1}^{N}\left(a^{(n)}\right)^{2} .
$$

Since $k_{i j}=k_{j i}$, only three independent scalars need to be determined. Rather than presenting these three scalars as $k_{11}, k_{22}$, and $k_{12}$, it is more useful to give the components of $k_{i j}$ along its major and minor axes and the angle $|\psi|$ between one of the principal axes and the $x_{1}$ axis. Thus the results of the computations will be presented in terms of $K_{m}, K_{d}$, and $\psi$ defined by

$$
\begin{aligned}
& K_{m}=\left(k_{11}+k_{22}\right) / 2, \\
& \tan 2 \psi=2 k_{12} /\left(k_{11}-k_{22}\right), \\
& K_{d}=\left|k_{12} \sin 2 \psi+\frac{1}{2}\left(\mathrm{k}_{11}-\mathrm{k}_{22}\right) \cos 2 \psi\right| .
\end{aligned}
$$

The components of $k_{i j}$ along its major and minor axes in the $x_{1}-x_{2}$ plane are given by

$$
\begin{aligned}
& k_{\text {maj }}=K_{m}+K_{d}, \\
& k_{\text {min }}=K_{m}-K_{d} .
\end{aligned}
$$

\section{RESULTS}

The method described in Sec. II was first applied to the case in which the $N$ particles within a unit cell were themselves arranged in a periodic array forming a unit cell whose dimension is an integral fraction of $h$. The infinite set of equations given by (13) and (14) was truncated to a finite set by retaining $A_{l m}$ with $l$ smaller than some integer $N_{p}$. In terms of multipole expansions, this means retaining all the terms with multipoles of order less than $2^{N p}$. This resulted in a total of $2 N_{p} N$ linear equations in an equal number of unknowns. The resulting equations were then solved and $K_{m}$, $K_{d}$, and $\psi$ were determined for the selected values of $\alpha$ and $\phi$. This procedure was repeated for larger values of $N_{p}$ until $K_{m}$ no longer changed appreciably. For $\phi=0.7$, the results converged for $N p \geqslant 8$. The convergence was even more rapid for smaller values of $\phi$. The calculated values of $K_{d}$ were less than $10^{-10}$ in all the cases examined and $K_{m}$ agreed with the results for the square arrays of cylinders reported by Perrins et $a l .{ }^{4}$ for the complete range of $\alpha$ and $\phi$. The calculations were made for $N$ equal to $1,4,9$, and 16 and no significant loss in the accuracy was observed as $N$ increased from 1 to 16.

Next, the calculations were performed with arrays in which the centers of the $N$ equal-sized particles were deter- 
mined by means of a computer program for generating random arrays similar to the Monte Carlo simulation techniques used for generating the hard-cylinder equilibrium distribution (see, for example, Chae et al. ${ }^{5}$ ). Briefly, the particles were first placed on a square lattice and then moved sequentially by a normally distributed random displacement. When all the particles were given a displacement once, one "random" configuration of the $N$ particles was generated. In this manner, about $10^{5}$ configurations were generated and the centers of the particles and the radial distribution function of the array were recorded at the interval of every $10^{4}$ configurations. A computer program by Venkatesan ${ }^{6}$ was employed for this purpose after a few modifications. The radial distribution function obtained by averaging over several (typically 6-11) configurations with $\phi=0.7$ is given in Table I for various $N$. Although the standard deviation (s.d.) among the arrays with $N=36$ is generally smaller than for those with $N=9$, it is seen that the changes in the radial distribution function as $N$ is increased from 9 to 36 are relatively insignificant. The radial distribution functions for the arrays with $\phi=0.1,0.3$, and 0.5 are given in Table II. Although the fluctuations in $g(r)$ at larger values of $r$ are somewhat larger in comparison with the values reported by Chae et al ${ }^{5}$ who calculated $g(r)$ using $N=208$, the agreement in $g(r / d)$ for $r / d$ close to unity is generally good.

The convergence of the numerical results for $k_{11}$ and $k_{22}$ for two arrays with $\phi=0.7$ and 0.3 is shown in Table III. The particles are infinitely conducting $(\alpha=\infty)$ and $N=16$ in these calculations. Also the total number of unknowns is $2 N_{p} N$. All the subsequent calculations were made with $N_{p}$ $=10$ for $\phi=0.7$ and $\phi=0.5$ and with $N_{p}=5$ for $\phi=0.3$ and $\phi=0.1$.

The calculated values of the mean and standard deviation (over eight to ten configurations) of $K_{m}, K_{d}$, and $|\psi|$ for the random arrays with $\phi=0.7$ are given in Table IV. Once again, no significant change is observed as $N$ is increased from 9 to 36 . Here $|\psi|$ was expected to vary uniformly between $0^{\circ}$ and $45^{\circ}$. A large standard deviation in $|\psi|$ in Table IV indicates that there was no significant bias in the orientation of the arrays generated in the study. Similarly a large standard deviation in $K_{d}$ indicates that the degree of anisotropy varied considerably among the arrays chosen for the computations. Since no significant change in $K_{m}$ was observed, all the subsequent calculations were made with

TABLE I. The radial distribution function $g(r / d), d$ being the diameter of the cylinders, for simulated arrays with $\phi=0.7$.

\begin{tabular}{lllll}
\hline \hline$r / d$ & \multicolumn{1}{c}{$N=36$} \\
mean \pm s.d. & $\begin{array}{c}N=25 \\
\text { mean } \pm \text { s.d. }\end{array}$ & $\begin{array}{c}N=16 \\
\text { mean } \pm \text { s.d. }\end{array}$ & $\begin{array}{c}N=9 \\
\text { mean } \pm \text { s.d. }\end{array}$ \\
\hline 1.1 & $3.41 \pm 0.13$ & $3.39 \pm 0.11$ & $3.45 \pm 0.11$ & $3.36 \pm 0.13$ \\
1.3 & $0.76 \pm 0.17$ & $0.9 \pm 0.17$ & $0.75 \pm 0.13$ & $0.55 \pm 0.22$ \\
1.5 & $0.56 \pm 0.1$ & $0.4 \pm 0.1$ & $0.46 \pm 0.13$ & $1.03 \pm 0.5$ \\
1.7 & $0.52 \pm 0.1$ & $0.52 \pm 0.1$ & $0.51 \pm 0.1$ & $0.44 \pm 0.12$ \\
1.9 & $0.94 \pm 0.38$ & $1.03 \pm 0.38$ & $0.95 \pm 0.23$ & $0.40 \pm 0.36$ \\
2.1 & $1.66 \pm 0.1$ & $1.65 \pm 0.1$ & $1.77 \pm 0.25$ & $1.75 \pm 0.59$ \\
2.3 & $1.17 \pm 0.26$ & $1.15 \pm 0.15$ & $1.08 \pm 0.21$ & $1.24 \pm 0.43$ \\
2.5 & $0.70 \pm 0.2$ & $0.71 \pm 0.15$ & $0.62 \pm 0.16$ & $0.99 \pm 0.17$ \\
2.7 & $0.71 \pm 0.2$ & $0.75 \pm 0.1$ & $0.76 \pm 0.11$ & $0.5 \pm 0.36$ \\
2.9 & $0.93 \pm 0.2$ & $0.97 \pm 0.12$ & $1.03 \pm 0.23$ & $0.64 \pm 0.22$ \\
& & & & \\
\hline \hline
\end{tabular}

TABLE II. The radial distribution functions for arrays with $\phi=0.1,0.3$, and 0.5 . The number of cylinders per unit cell is 16 in all cases.

\begin{tabular}{cccc}
\hline \hline$r / d$ & $\phi=0.1$ & $\phi=0.3$ & $\phi=0.5$ \\
\hline 1.1 & 1.54 & 1.74 & 2.77 \\
1.3 & 1.30 & 1.59 & 0.77 \\
1.5 & 1.21 & 1.25 & 0.54 \\
1.7 & 0.99 & 0.98 & 0.83 \\
1.9 & 0.73 & 0.89 & 1.20 \\
2.1 & 0.68 & 0.93 & 1.29 \\
2.3 & 1.02 & 1.03 & 0.83 \\
2.5 & 1.08 & 1.04 & 0.83 \\
2.7 & 0.65 & 1.01 & 1.04 \\
2.9 & 1.30 & 0.99 & 1.12 \\
\hline \hline
\end{tabular}

$N=16$. The results for $\phi=0.5,0.3$, and 0.1 are also presented in Table IV. This constitutes the principal results of the present study. In Sec. IV A $K_{m}$ as a function of $\alpha$ for a few selected values of $\phi$ is presented.

\section{COMPARISON WITH OTHER METHODS}

One possible application of the results presented in Sec. III is to provide estimates of the effective thermal conductivity of the random arrays that can be used to assess the range of validity or the usefulness of various methods suggested in the literature to predict the effective conductivity, at least as far as their applicability to the composites with cylindrical inclusions is concerned. We shall compare the results in detail using three different approaches.

\section{A. Bounds}

The only known exact results for the effective conductivity to date are for the square and hexagonal arrays of cylinders. ${ }^{4} \mathrm{~A}$ detailed comparison of these exact results with various bounds on the thermal conductivity was made by McPhedran and Milton. ${ }^{7}$ In this section, we shall compare various bounds with the results obtained in Sec. III. Hashin and Shtrikman ${ }^{8}$ have shown that the effective conductivity of isotropic (in the $x_{1}-x_{2}$ plane) arrays of parallel cylinders is bounded by $K_{l}$ and $K_{u}$ given by

$$
\begin{aligned}
& K_{l}=1+\phi /[1 /(\alpha-1)+(1-\phi) / 2], \\
& K_{u}=\alpha+(1-\phi) /[1 /(1-\alpha)+\phi / 2 \alpha],
\end{aligned}
$$

TABLE III. Convergence of the numerical results for $k_{11}$ and $k_{22}$ for two arrays with $\phi=0.7$ and 0.3 . The conductivity of the particles is infinite, $N$, the number of particles per unit cell is 16 , and the total number of unknowns is $2 N_{p} N$.

\begin{tabular}{cccccc}
\hline \hline$N_{p}$ & \multicolumn{2}{c}{$\phi=0.7$} & & \multicolumn{2}{c}{$\phi=0.3$} \\
& $k_{11}$ & $k_{22}$ & & $k_{11}$ & $k_{22}$ \\
\cline { 2 - 3 } \cline { 5 - 6 } 11 & 7.66 & 7.92 & & $\ldots$ & $\ldots$ \\
10 & 7.64 & 7.91 & & $\ldots$ & $\ldots$ \\
9 & 7.61 & 7.87 & & $\ldots$ & $\ldots$ \\
8 & 7.56 & 7.83 & & 2.05 & 1.98 \\
7 & 7.51 & 7.78 & & 2.05 & 1.98 \\
6 & 7.39 & 7.70 & & 2.05 & 1.98 \\
5 & 7.25 & 7.56 & & 2.05 & 1.98 \\
4 & $\cdots$ & $\cdots$ & 2.04 & 1.97 \\
\hline \hline
\end{tabular}


TABLE IV. $K_{m}, K_{d}$ and $|\psi|$ [see (22)] for the arrays of infinitely conducting $(\alpha=\infty)$ cylinders.

\begin{tabular}{|c|c|c|c|c|}
\hline$N$ & $\begin{array}{c}K_{m} \\
(\text { mean } \pm \text { s.d. })\end{array}$ & $\begin{array}{c}K_{d} \\
\text { (mean } \pm \text { s.d.) }\end{array}$ & $\begin{array}{c}|\psi| \\
\text { (mean } \pm \text { s.d.) }\end{array}$ & $\phi$ \\
\hline 9 & \pm 0.056 & $0.70 \pm 0.33$ & $19.4 \pm 13.8$ & \\
\hline 16 & \pm 0.34 & $0.67 \pm 0.40$ & $15.8 \pm 7.4$ & \\
\hline 25 & \pm 0.25 & $0.83 \pm 0.48$ & $18.8 \pm 12.1$ & 0.7 \\
\hline 36 & $7.8 \pm 0.34$ & $0.37 \pm 0.24$ & $21.7 \pm 0.34$ & \\
\hline 16 & $3.40 \pm 0.06$ & $0.22 \pm 0.26$ & $23.3 \pm 12.2$ & 0.5 \\
\hline 16 & $1.97 \pm 0.03$ & $0.07 \pm 0.05$ & $36 \pm 6.7$ & 0.3 \\
\hline 16 & $1.230 \pm 0.002$ & $0.020 \pm 0.027$ & $22.9 \pm 12.4$ & 0.1 \\
\hline
\end{tabular}

where $K$ 's are nondimensionalized by the conductivity of the matrix. As $\alpha \rightarrow \infty$, i.e., when the conductivity of the cylinders is very large, $K_{u}$ approaches infinity for any nonzero $\phi$, whereas $K_{l}$ approaches a constant equal to $(1+\phi) /$ $(1-\phi)$. Since $K_{t}$ and $K_{u}$ are equal to each other up to $O\left(\beta^{2}\right)$, where $\beta \equiv(\alpha-1) /(\alpha+1)$, the bounds given by (24) are sometimes referred to as the second-order bounds. A comparison of $K_{l}$ with $K_{m}$ calculated in Sec. III is shown in Table $\mathrm{V}$, where it is seen that the difference between $K_{m}$ and $K_{l}$ at $\phi=0.7$ and $\alpha=\infty$ is approximately $30 \%$, the difference being lower for smaller values of $\alpha$ or $\phi$.

\section{The third-order bounds and the evaluation of Milton's number}

Hashin and Shtrikman have shown that their bounds are the best possible ones when the only known information about the composite medium is the volume fractions and the conductivities of the individual phases. In order to obtain bounds in which the difference between $K_{u}$ and $K_{t}$ is smaller, additional information about the geometry of the composite medium must be given. Beran ${ }^{9}$ and Silnutzer ${ }^{10}$ have given the third-order bounds, i.e., bounds in which $K_{u}$ and $K_{l}$ coincide to $O\left(\beta^{3}\right)$, which require, in addition to $\phi$ and $\alpha$, a parameter related to the three-point correlation function of the geometry of the composite medium. There are several other equivalent expressions of the third-order bounds, all of which require the three-point correlation functions. The evaluation of the parameter appearing in the third-order bounds has been the subject of many articles by Torquato and co-workers (see, for example, Refs. 11-13) in recent years. These investigators have simplified a key integral appearing in the expression for the third-order bounds and then by invoking a superposition approximation according to which a three-point correlation function is expressed as a product of two-point correlation functions, a parameter

TABLE V. The Hashin-Shtrikman (second-order) and higher-order lower bounds on the effective conductivity of random arrays of infinitely conducting $(\alpha=\infty)$ cylinders. The tenth-order bound coincides with the exact results for these arrays.

\begin{tabular}{cccc}
\hline \hline$\phi$ & Second-order & Fourth-order & Tenth-order \\
\hline 0.7 & 5.66 & 6.75 & 7.65 \\
0.5 & 3.00 & 3.25 & 3.36 \\
0.3 & 1.86 & 1.98 & 2.02 \\
0.1 & 1.222 & 1.226 & 1.227 \\
\hline
\end{tabular}

known as Milton's number $\zeta$ is evaluated for various geometrical configurations of the composite medium. Since the two third-order bounds coincide to $O\left(\beta^{3}\right)$, it is not surprising that this parameter is related to the coefficients $Q_{n}$ in the expansion of $K_{m}$ in powers of $\beta$ defined by

$$
K_{m}=1+\sum_{n=1}^{\infty} Q_{n} \frac{\beta^{n}}{n !}
$$

The exact relationship between $Q_{3}$ and $\zeta$ for the array of cylinders, i.e., the two-dimensional arrays, is

$$
\zeta=[1 / 2 \phi(1-\phi)]\left(\frac{1}{6} Q_{3}-2 \phi^{2}\right) \text {. }
$$

This connection between $Q_{3}$ and $\zeta$ provides us with an alternate method to evaluate the third-order bounds rigorously. Thus we first expand each of the coefficients $A_{l m}$ in (13) and (14) in powers of $\beta$ and obtain a recursion relation among the coefficients in the expansion of $A_{l m}$ in powers of $\beta$. The series for $A_{10}$ is then related to $Q_{n}$ in (25) via (19). Then, on using (26), $\xi$ could be computed for the cases of mean temperature gradients in the $x_{1}$ and $x_{2}$ directions separately. An arithmetic average of $\zeta$ computed from these two cases should yield a reasonable estimate of Milton's number. The results are shown in Table VI, where the mean and the standard deviation of $\left(\xi_{11}+\zeta_{22}\right) / 2$ and $\left|\zeta_{11}-\zeta_{22}\right| / 2$ are given for several configurations with $\phi$ ranging from 0.1 to 0.7 . The convergence of Milton's number with $N_{p}$ was found to be very rapid. Although various arrays chosen for the calculation of Milton's number were anisotropic to different extents, the mean $\left(\zeta_{11}+\zeta_{22}\right) / 2$ showed a relatively minor variation among the arrays generated. Torquato and Lado ${ }^{13}$ have recently calculated Milton's number for similar random arrays by invoking the approximate method described earlier. Their estimates of Milton's parameter are also given in Table VI. We see that the agreement between the two methods is very good for $\phi$ less than 0.3 . This is not surprising, as the superposition approximation invoked by Torquato and Lado is exact to $O\left(\phi^{2}\right)$. At $\phi=0.5$, however, their results are about $40 \%$ higher. Whether this difference at higher values of $\phi$ is a consequence of the superposition approximation employed by these authors or a consequence of using only 16-32 particles per unit cell in our simulations is difficult to assess at present. It is known that the effective conductivity of the arrays of cylinders is a relatively insensitive function of the packing and the geometrical parameter $\zeta$. Thus even though the effective conductivity of the random arrays appears to have approached a nearly constant value as $N$ is increased to 36 , it is quite possible that a much larger value of $N$ may by necessary in order to obtain accurate

TABLE VI. Results for the third-order bounds.

\begin{tabular}{ccccc}
\hline \hline$\phi$ & $N$ & $\begin{array}{c}\frac{1}{2}\left(\zeta_{11}+\zeta_{22}\right) \\
\text { mean } \pm \text { s.d. }\end{array}$ & $\begin{array}{c}\frac{1}{2}\left|\zeta_{11}-\zeta_{22}\right| \\
\text { mean } \pm \text { s.d. }\end{array}$ & $\begin{array}{c}\text { Torquato- } \\
\text { Lado }^{\mathrm{a}}\end{array}$ \\
\hline 0.7 & 36 & $0.20 \pm 0.016$ & $0.06 \pm 0.04$ & $\cdots$ \\
0.5 & 16 & $0.118 \pm 0.018$ & $0.020 \pm 0.009$ & 0.165 \\
0.3 & 16 & $0.091 \pm 0.019$ & $0.010 \pm 0.010$ & 0.092 \\
0.1 & 16 & $0.031 \pm 0.008$ & $0.005 \pm 0.003$ & 0.032 \\
\hline \hline
\end{tabular}

"See Ref. 13. 
estimates of $\zeta$. We understand that Torquato and co-workers are currently undertaking rigorous calculations of $\xi$ for arrays with a much larger $N$. Their calculations may provide the definitive answer in assessing the accuracy of the superposition approximation.

\section{Higher-order bounds}

Milton $^{14}$ has shown how the higher-order bounds are related to $Q_{n}$. Thus for example, a lower bound of order $2 N_{b}$ is an $\left[\mathrm{N}_{b} / \mathrm{N}_{b}\right.$ ] Padé approximant of the series (25) truncated to $n \leqslant 2 N_{b}$. Similarly an upper bound of order $2 N_{b}$ is an $\left[N_{b}+1 / N_{b}+1\right]$ Padé approximant of (25) with the first $2 N_{b}$ coefficients retained in it and with two additional constraints that $K_{u}=\infty$ for $\alpha=\infty$ and $K_{u}=0$ for $\alpha=0$. Since it is rather straightforward to evaluate the coefficients of $Q_{n}$ by the method described in Sec. IV A 1, it is possible to compute $K_{l}$ or $K_{u}$ to arbitrarily high orders in $\beta$. In Table V, the results for the second-, fourth-, and tenth-order lower bounds are given for various $\phi$ when $\alpha=\infty$. These results are obtained for only one representative array for each value of $\phi$ and thus the conductivity obtained from the tenth-order bound is slightly different from the mean over several configurations reported in Table IV. Because of the anisotropy of the chosen array, $Q_{n}$ computed for two different orthogonal directions were different from each other and an arithmetic mean of $Q_{n}$ for each $n$ was employed in determining the bounds. A separate calculation of $K_{m}$ using the direct method described in Sec. III showed that the results for the tenthorder lower bounds coincided with the exact value of $K_{m}$. We see that the fourth-order lower bound is only $13 \%$ lower than the exact value of the effective conductivity at $\alpha=\infty$ and $\phi=0.7$, and that the deviation between the two is much smaller at smaller values of $\phi$.

The evaluation of the effective thermal conductivity via the determination of the higher-order lower bounds provides an efficient numerical method to determine the effective conductivity as it does not require solving a system of linear equations. In fact, this was exploited by the present investigators ${ }^{15}$ recently to estimate the effective conductivity of the composites containing random arrays of spherical particles. Once $Q_{n}$ is determined, it is computationally far more efficient to compute $K_{m}$ as a function of $\alpha$. Figure 1 shows $K_{m}$ as

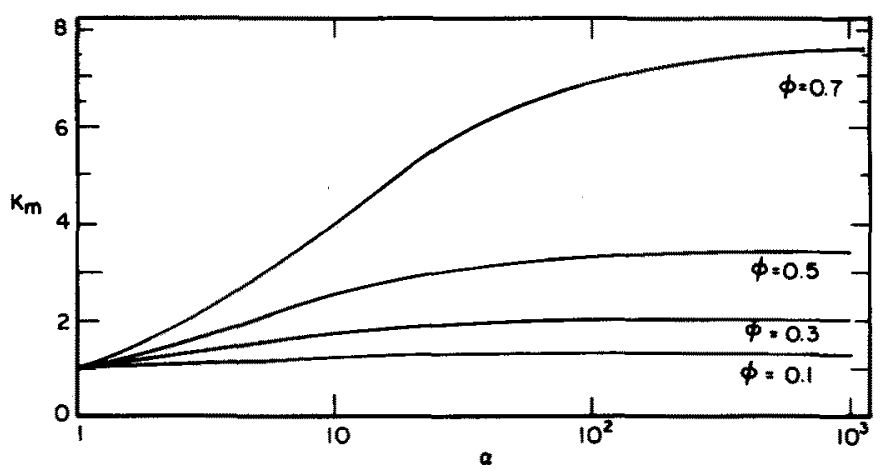

FIG. 1. The effective thermal conductivity as a function of $\alpha$ for various values of $\phi$. a function of $\alpha$ for various $\phi$ for the arrays used in computing the bounds in Table V. Although these estimates are for only one configuration for each $\phi$, the mean over several configurations with the same $\phi$ is expected to be quantitatively similar.

\section{B. Approximate methods}

These are methods in which rather than solving for the temperature field in a two-phase medium in which the dispersed phase is distributed homogeneously throughout the composite medium, one determines the temperature field in a "model" of the composite medium in which a particle is placed in a medium whose properties are suitably defined. Probably the most well-known example of such methods is the self-consistent scheme according to which the properties of the medium are defined as

$$
k_{m}(r)= \begin{cases}1, & \text { if } r \leqslant \phi^{-1 / 2}, \\ K_{m}, & \text { if } r>\phi^{-1 / 2},\end{cases}
$$

where $K_{m}$ is the conductivity to be determined in a selfconsistent manner by first solving (27) in terms of $K_{m}$ and then determining the strength of thermal dipole at the origin and relating it to $K_{m}$ [cf. (31) below]. This scheme yields an estimate of $K_{m}$ that coincides with the Hashin-Shtrikman lower bound, which was shown to be approximately $30 \%$ lower than the actual value of $K_{m}$ at $\phi=0.7$ and $\alpha=\infty$. The estimate from this method is very good at lower values of $\phi$ and $\alpha$.

Recently Chang et al. ${ }^{16,17}$ have presented two approximate methods which they referred to as methods $A$ and $B$. Of these two, method B was originally suggested by Buyevich and Korneev. ${ }^{18}$ Both of these methods are similar to the selfconsistent scheme except that, in addition to $\phi$ and $\alpha$, they also require the knowledge of the probability density function $\rho(r)$ of the dispersed phase in the vicinity of any representative particle. For the case of two-dimensional arrays of interest to us here, $\rho(r)$ and $g(r)$ are related by

$$
\rho(r)=\frac{2 \phi}{\pi a^{2}} \int_{r-a}^{r+a} g(r) \cdot R \cos ^{-1} \frac{R^{2}+r^{2}-a^{2}}{2 R r} d R
$$

and $\rho(r) \rightarrow \phi$ as $r \rightarrow \infty$.

In method $A$, the temperature field around a cylinder situated at the origin satisfies

$$
\begin{aligned}
& \nabla^{2} T=0, \quad r<1, \\
& \nabla \cdot\left[k_{m}(r) \nabla T\right]=0, \quad r \geqslant 1, \\
& T \rightarrow r \cos \theta, \quad \text { as } r \rightarrow \infty, \\
& \alpha\left(\frac{\partial T}{\partial r}\right)_{-}=\left(\frac{\partial T}{\partial r}\right)_{+}, \quad r=1,
\end{aligned}
$$

in addition to the continuity of the temperature at the surface of the cylinder. The radius of the cylinder is taken to be unity. The conductivity of the medium is given by

$$
k_{m}(r)=1+\left(K_{m}-1\right) \rho(r) / \phi,
$$

where $K_{m}$ is, once again, the effective conductivity to be determined. We note that, in particular, if we substitute $\rho(r)=0$ for $r<\phi^{-1 / 2}$ and $\rho(r)=\phi$ for $r>\phi^{-1 / 2}$ in (30), method $A$ reduces to the original self-consistent scheme. In this respect, method A may be regarded as a modification of 
the self-consistent scheme. Once the temperature field is determined, the estimate of $K_{m}$ is obtained from

$$
K_{m}=1+(\alpha-1)\left(\frac{\partial T}{\partial x_{1}}\right)_{0},
$$

where $\left(\partial T / \partial x_{1}\right)_{0}$ is the temperature gradient at the center of the particle.

Method B is similar to method A. Here, one solves for the disturbance temperature $t$ instead of $T$, i.e., one solves

$$
\begin{aligned}
& T=x_{1}+t, \\
& \nabla \cdot\left[k_{m}(r) \nabla t\right]=0, \quad r \geqslant 1, \\
& \nabla^{2} t=0, \quad r<1, \\
& \alpha\left[x_{1}+\left(\frac{\partial t}{\partial r}\right)_{-}\right]=K_{m} x_{1}+\left(\frac{\partial t}{\partial r}\right)_{+}, \quad \text { at } r=1, \\
& t \rightarrow 0, \text { as } r \rightarrow \infty,
\end{aligned}
$$

where, once again, $k_{m}(r)$ is given by (30). Chang et al. ${ }^{17}$ showed that, as $\phi$ and $\beta$ approach zero, the estimates of $K_{m}$ obtained by method $A$ become exact, and thus provided a rigorous justification for method A. Method B, however, which does not yield exact estimates for $K_{m}$ at small $\phi$ and $\beta$, was shown to yield estimates of the effective thermal conductivity of the random arrays of spheres which were in better agreement with the corresponding experimental results at large values of $\phi$ and $\alpha$.

Using the radial distribution functions of the random arrays simulated in the present study, $\rho(r)$ was computed for each $\phi$ and, on solving (29)-(32), the estimates of the effective conductivity on the random arrays of cylinders were obtained. These are compared with the corresponding values obtained from the exact method of Sec. III in Table VII. Similar calculations for the square and hexagonal arrays of cylinders were also made and the values for these arrays are also given in Table VII. The following observations may be made.

(i) The self-consistent scheme, which does not require any information on the geometry of the two-phase medium other than $\phi$, always underestimates $K_{m}$. This is not surprising, since, as mentioned earlier, the estimate obtained by this scheme coincides with the Hashin-Shtrikman lower bound.

(ii) The estimates obtained from method $\mathrm{A}$ are better than those obtained from method B for almost the whole range of $\phi$ and $\alpha$. In particular, method $B$ always overestimates $K_{m}$ and fails severely at large $\phi$. This is in contrast to the arrays of spherical particles for which method $B$ gave better estimates of $K_{m} \cdot{ }^{17}$

(iii) Method A gives better estimates than the self-consistent scheme in all the cases given in Table VII with an exception for a hexagonal array with $\phi=0.7$ and $\phi=0.85$. The particles are more effectively separated from each other in the case of a hexagonal array than for the square and random arrays and, hence it is not surprising that the selfconsistent scheme, which assumes $k_{m}=1$ for $r<\phi^{-1 / 2}$, yields a relatively better estimate $K_{m}$. A closer examination of the results presented in Table VII, however, reveals that method A does not seem to yield estimates consistent with the changes in the radial distribution function. For example, the estimates from method A for the square, random, and
TABLE VII. Comparison of the effective conductivity of the random (RA), square (SA), and hexagonal (HA) arrays of infinitely conducting $(\alpha=\infty)$ cylinders estimated via the exact, self-consistent scheme (SC), and methods A and B (MA and MB).

\begin{tabular}{lccccc}
\hline \hline$\phi$ & Array & Exact & SC & MA & MB \\
\hline 0.85 & HA & 15.67 & 12.73 & 10.9 & $>10^{2}$ \\
0.75 & SA & 12.75 & 7.0 & 8.1 & $>10^{2}$ \\
0.7 & SA & 7.43 & 5.66 & 7.2 & 24 \\
0.7 & HA & 5.81 & 5.66 & 8.2 & 71 \\
0.7 & RA & $7.9 \pm 0.3$ & 5.66 & 6.1 & 31 \\
0.5 & RA & $3.4 \pm 0.06$ & 3.00 & 3.3 & 5.1 \\
0.5 & SA & 3.08 & 3.00 & 2.8 & 3.5 \\
0.3 & RA & $1.97 \pm 0.03$ & 1.86 & 1.97 & 2.04 \\
0.3 & SA & 1.86 & 1.86 & 1.81 & 1.91 \\
0.1 & RA & 1.23 & 1.22 & 1.23 & 1.24 \\
\hline \hline
\end{tabular}

hexagonal arrays, all with $\phi=0.7$, are, respectively, 7.2,6.1, and 8.2 compared with the exact values for these arrays of $7.43,7.9 \pm 0.3$, and 5.81. In other words, although the method requires an additional piece of information, viz., $\rho(r)$ of the composite medium, over that required by the self-consistent scheme, it does not seem to utilize it adequately, even in the qualitative sense.

\section{Asymptotic methods}

Peterson and Hermans ${ }^{19}$ have obtained an expression for the effective thermal conductivity of the random arrays of cylinders with $\phi \ll 1$ by taking into account the pairwise interactions using a technique similar to the one employed later by Jeffrey ${ }^{20}$ who treated the analogous case for the arrays of spheres. In both of these studies, the random arrays are defined by

$$
g(r)= \begin{cases}0, & r<d, \\ 1, & r \geqslant d,\end{cases}
$$

$d$ being the diameter of the particles. Peterson and Hermans showed that for such arrays $K_{m}$ is given by

$$
K_{m}=1+2 \beta \phi+2 \beta^{2} K(\beta) \phi^{2}+O\left(\phi^{3}\right),
$$
where

$$
\begin{aligned}
& K(\beta)=1+\frac{1}{3}\left(1+0.0835 \beta^{2}+0.0194 \beta^{2}\right. \\
& \left.+0.00505 \beta^{6}+\cdots\right) .
\end{aligned}
$$

For the infinitely conducting cylinders $(\beta=1), K(1)$ is approximately 1.37 and (34) gives the estimates of $K_{m}$ at $\phi=0.1,0.3$, and 0.5 to be, respectively, $1.227,1.85$, and 2.69 , which on comparison with the exact results of Sec. III (cf. Table IV), viz., $K_{m}$ equal to $1.230 \pm 0.002,1.97 \pm 0.03$, and $3.40 \pm 0.06$, suggests that (34) gives a reasonably accurate estimate up to $\phi=0.3$. It is interesting to note that a Padé approximant of (34), viz.,

$$
K_{m}=\{1+\beta[2-K(\beta)] \phi\} /[1-\beta K(\beta) \phi],
$$

which yields $K_{m}$ equal to 4.2 for $\beta=1$ and $\phi=0.5$, does not give any better estimate of $K_{m}$ as compared with (34) at higher $\phi$.

If the particle conductivity is very large and the gap or the minimum distance between the neighboring particles is small compared to the diameter of the particles, then the 
heat flux in the gaps between the pairs of two nearly touching particles with unequal temperatures tends to dominate the overall heat flux and hence the effective conductivity of the medium. For a special case of random arrays with large $\phi$ in which the gap width between the neighboring particles is nearly equal for all of the particles, it is possible to obtain an estimate of $K_{m}$ at large $\phi$ and $\alpha$. Batchelor and O'Brien ${ }^{21}$ have derived expressions for $K_{m}$ applicable to such random arrays for the case of spherical particles. A similar treatment for the cylindrical particles yields ${ }^{22}$

$$
K_{m}=\left\{\begin{array}{rr}
(1 / \sqrt{2}) Z \phi_{t}\left[1-\left(\phi / \phi_{t}\right)^{1 / 2}\right]^{-1 / 2}+K_{1}, \\
\alpha=\infty, \quad \phi \rightarrow \phi_{t}, \\
\frac{1}{2} Z \phi_{t} \alpha / \log \alpha+K_{2}, \quad \alpha \rightarrow \infty, \quad \phi=\phi_{t},
\end{array}\right.
$$

where $Z$ is the coordination number of the array, i.e., the average number of nearest neighbors per particle, $\phi_{t}$ corresponds to the area fraction of the particle in the closely packed configuration, and $K_{1}$ and $K_{2}$ are $O(1)$ constants. This special class of random arrays may be simulated numerically via a two step procedure. In the first step, the process of dropping a large number of equal-sized particles in a container is simulated. This results in a closely packed random array with an area fraction $\phi_{t}$. Several investigators have simulated such arrays and their results are summarized by Berryman. ${ }^{23}$ According to these investigations, $\phi_{t}$ is in the range $0.81-0.89$ with most studies quoting a value of approximately 0.82 . We have also simulated such arrays recently with as many as 1600 cylinders in a container and found $\phi_{t}$ to be approximately 0.824 . The average coordination number $Z$ was found to be 4.2. In the second step, the centers of the cylinders generated by the above procedure are assumed to remain fixed while the diameter of each of the particles is reduced by a fraction $\chi$. This results in an array whose area fraction $\phi$ is related to $\chi$ by

$$
\chi=\left(\phi / \phi_{t}\right)^{1 / 2}
$$

The particles in such random arrays have a nearly uniform gap-size distribution while the random arrays described in Sec. III need not have a uniform gap-size distribution. In fact, the simulation procedure of Sec. III does not exclude the possibility of "cluster" formations within the random array. If the "clusters" are present, the conduction of heat is not uniformly distributed among the particles and, consequently, the effective conductivity computed in Sec. III need not show the asymptotic behavior in accordance with (36) at large $\phi$ and $\alpha$. Nevertheless it is interesting to compare the estimates obtained from (36) with those of Sec. III. For the purpose of comparison we choose $K_{1}$ to be such that $K_{m} \rightarrow 1$ as $\phi \rightarrow 0$, i.e., for the case of perfectly conducting particles, we rewrite (36) as

$$
\begin{aligned}
K_{m}= & 1+(1 / \sqrt{2}) Z \phi_{t}\left\{\left[1-\left(\phi / \phi_{t}\right)^{1 / 2}\right]^{-1 / 2}-1\right\} \\
& (\alpha=\infty) .
\end{aligned}
$$

A comparison of $K_{m}$ estimated from (38) with $Z=4.2$ and $\phi_{t}=0.82$ with the results obtained in Sec. III is given in Table VIII. Formula (38) provides a reasonably good estimate for all the quoted values of $\phi(0.1 \leqslant \phi \leqslant 0.7)$ with the maximum deviation of approximately $20 \%$ occurring at $\phi=0.3$. A similar comparison between the exact results for the square and hexagonal arrays as obtained by Perrins $e t$ $a l .^{4}$ with formula (38) is also given in Table VIII, where the values of $Z$ and $\phi_{t}$ are chosen to be, respectively, 4 and $\pi /$ $4=0.785$ for the square and 6 and $\pi / 2 \sqrt{ } 3=0.907$ for the hexagonal arrays. The approximate formula (38) provides reasonably good estimates in these cases also.

\section{v. CONCLUSIONS}

A numerical method is developed for solving the heat conduction equation in two-phase composite media consisting of suspensions of long circular cylinders. This method is applied to the spatially periodic media whose unit cells contain $N$ cylinders arranged randomly and the effective thermal conductivity of the composite media is determined for several configurations of the $N$ cylinders. The effect of varying $N$ from $N=9$ to $N=36$ on the effective thermal conductivity (averaged over several configurations with the same $N$ ) is found to be insignificant and hence it is believed that the results presented here are applicable to the random arrays of cylinders with $N=\infty$. Also, in view of the relatively small variations in the effective thermal conductivity among various configurations with the same $\phi$, the conductivity appears to be a relatively weak function of the detailed arrangement of the particles and this makes it meaningful to relate the effective conductivity to the minimal information regarding the geometry such as the area or volume fraction and the radial distribution function of the array, at least for $\phi \leqslant 0.7$. The results obtained via the exact method were compared with various approximate theories proposed in the literature to estimate the effective conductivity. The HashinShtrikman lower bound, or equivalently, the self-consistent scheme which only requires knowledge of $\phi$ and $\alpha$ provides a very satisfactory estimate of $K_{m}$ (accurate to within 30\%) for all $\alpha$ and $\phi \leqslant 0.3$. Formula (38) based on a high area fraction asymptote with $\alpha=\infty$ provides fairly accurate estimates of $K_{m}$ for the complete range of $\phi$. One of the two approximate methods proposed recently by Chang et al. ${ }^{17}$ also appears to yield very accurate estimates of the effective conductivity.

Although the method developed here was employed for determining the effective conductivity of cylindrical particles satisfying the classical boundary conditions of the continuity of the temperature and normal flux, the method is

TABLE VIII. Comparison of the exact results for the high area fraction formula (38).

\begin{tabular}{ccccccc}
\hline \hline \multicolumn{3}{c}{ Random array } & \multicolumn{3}{c}{ Square array } & \multicolumn{2}{c}{ Hexagonal array } \\
$\phi$ & exact & $(38)$ & exact $^{\mathrm{a}}$ & $(38)$ & exact $^{\mathrm{a}}$ & $(38)$ \\
& & & & & & \\
\hline 0.7 & $7.8 \pm 0.3$ & 7.4 & 7.43 & 8.2 & 5.81 & 8.24 \\
0.5 & $3.4 \pm 0.6$ & 3.8 & 3.08 & 3.72 & 3.00 & 4.73 \\
0.3 & $1.97 \pm 0.03$ & 2.44 & 1.86 & 2.37 & 1.86 & 3.06 \\
0.1 & $1.23 \pm 0.002$ & 1.59 & 1.22 & 1.55 & 1.22 & 1.86 \\
& & & & & & \\
\hline \hline
\end{tabular}

"Taken from Ref. 4. 
equally suitable to treat more complex boundary conditions including those that arise in the case of reacting or adsorbing particles, or particles with finite "skin" resistance. The method could also be used to determine the detailed temperature field around a "cloud" of a finite number of particles immersed in an infinite medium or to more complex geometries involving plane walls for which, by devising a suitable "image" system, the problem can be reduced, once again, to the study of temperature field around the "cloud" of particles.

\section{APPENDIX: FORMULAS FOR CALCULATING THE DERIVATIVES OF $\boldsymbol{s}_{\mathbf{1}}$}

For smaller $r(r<h / 2)$, the derivatives of $S_{1}(r)$ can be computed by the termwise differentiation of $(8)$. The required formulas are easily derived to be

$$
\begin{aligned}
\frac{\partial^{n+m}}{\partial x_{1}^{m} \partial x_{2}^{n}} & (\log r) \\
= & (-1)^{m-1}(m+n-1) ! r^{-n-m} \\
& \times\left\{\begin{array}{l}
(-1)^{n / 2} \cos (m+n) \theta, \quad n=\text { even, } \\
(-1)^{(n+1) / 2} \sin (m+n) \theta, \quad n=\text { odd, }
\end{array}\right. \\
\frac{\partial^{m+n}}{\partial x_{1}^{m} \partial x_{2}^{n}}\left(r^{p} \cos \theta\right) & \\
= & \frac{p !}{(p-n-m) !} r^{p-m-n} \\
& \times\left\{\begin{array}{l}
(-1)^{n / 2} \cos (p-m-n) \theta, \quad n=\text { even, } \\
(-1)^{(n+1) / 2} \sin (p-m-n) \theta, \quad n=\text { odd }
\end{array}\right.
\end{aligned}
$$

For a higher-order differentiation and for $r>h / 2$, the series computed from the termwise differentiation does not converge. For such situations, it is desirable to start with an integral representation of $S_{1}$,

$$
S_{1}(r)=\sum_{\mathbf{r}_{\mathbf{L}}}\left(-2 \log \left(\mathbf{r}-\mathbf{r}_{\mathbf{L}}\right)+\int_{\tau_{\mathbf{L}}} 2 \log \left(\mathbf{r}-\mathbf{r}^{\prime}\right) d \mathbf{r}^{\prime}\right),
$$

in which $\tau_{\mathbf{L}}$ is the unit cell containing $\mathbf{r}_{\mathbf{L}}$. The derivative of the second term in the above expression vanishes whereby

$$
\begin{aligned}
\frac{\partial^{n+m}}{\partial x_{1}^{m} \partial x_{2}^{n}} S_{1}(\mathbf{r}) & \\
= & \sum_{\mathbf{r}_{\mathbf{L}}} 2(-1)^{m}(m+n-1) ! s^{-n-m} \\
& \times \begin{cases}(-1)^{n / 2} \cos (m+n) \theta, \quad n=\text { even, } \\
(-1)^{(n+1) / 2} \sin (m+n) \theta, \quad n=\text { odd, },\end{cases}
\end{aligned}
$$

where $s$ and $\theta$ are measured with respect to $\mathbf{r}_{\mathbf{L}}$ as the origin.

'H. Hasimoto, J. Fluid Mech. 5, 317 (1959).

${ }^{2}$ A. S. Sangani, Ph.D. thesis, Stanford University, 1982.

${ }^{3}$ R. S. Rayleigh, Philos. Mag. 34, 481 (1892).

${ }^{4}$ W. T. Perrins, D. R. McKenzie, and R. C. McPhedran, Proc. R. Soc. London Ser. A 369, 207 (1979).

${ }^{5}$ D. G. Chae, F. H. Ree, and T. Ree, J. Chem. Phys. 50, 1581 (1969).

${ }^{6} \mathrm{M}$. Venkatesan, Ph.D. thesis, Rensselaer Polytechnic Institute, 1985.

${ }^{7}$ R. C. McPhedran and G. W. Milton, Appl. Phys. A26, 207 (1981).

${ }^{8}$ Z. Hashin and S. Shtrikman, J. Appl. Phys. A33, 3125 (1962).

${ }^{9}$ M. Beran, Nuovo Cimento 38, 771 (1965).

${ }^{10}$ N. Silnutzer, Ph.D. thesis, University of Pennsylvania, 1972.

"S. Torquato and F. Lado, Phys. Rev. B 33, 6428 (1986).

${ }^{12}$ S. Torquato and G. Stell, J. Chem. Phys. 77, 2071 (1982).

${ }^{13}$ S. Torquato and F. Lado, Proc. R. Soc. London 417, 59 (1988).

${ }^{14}$ G. W. Milton, Phys. Rev. Lett. 46, 542 (1981).

${ }^{15}$ A. S. Sangani and C. Yao, J. Appl. Phys. 63, 1334 (1988).

${ }^{16}$ E. Chang and A. Acrivos, J. Appl. Phys. 59, 3375 (1986).

${ }^{17}$ E. Chang, B. S. Yendler, and A. Acrivos, in Advances in Multiphase Flow and Related Problems, edited by G. Papanicolaou (SIAM, Philadelphia, Pennsylvania, 1986).

${ }^{18}$ Y. A. Buyevich and Y. A. Korneev, J. Eng. Phys. 31, 607 (1976).

${ }^{19}$ J. M. Peterson and J. J. Hermans, J. Compos. Mater. 3, 338 (1969).

${ }^{20}$ D. J. Jeffrey, Proc. R. Soc. London Ser. A 335, 355 (1973).

${ }^{2}$ 'G. K. Batchelor and R. W. O'Brien, Proc. R. Soc. London Ser. A 355, 313 (1977).

${ }^{22}$ R. W. O'Brien, Ph.D. thesis, Cambridge University, 1979.

${ }^{23}$ J. Berryman, Phys. Rev. A 27, 1053 (1983). 